\title{
On the uniform asymptotic expansion of the Legendre functions.
}

\author{
Nail R. Khusnutdinov * \\ Department of Physics, Kazan State Pedagogical University, Mezhlauk 1, Kazan, 420021, Russia
}

\begin{abstract}
An uniform expansion of the Legendre functions of large indices are considered by using the WKB approach. We obtain the recurrent formula for the coefficients of uniform expansion and compare them with the uniform expansion of the Bessel function.
\end{abstract}

PACS numbers: 02.30.Gp,02.30.Mv,04.62.+v

\section{INTRODUCTION}

An uniform expansion of special functions is very useful representation of them which is used in many branches of science. It is well-known, for example, the Debay uniform expansion of the Bessel functions [1]. To obtain the uniform expansion one usually uses the complicate calculations which exploit an contour integral representation of function (see for example [4]). In this paper we use the WKB approach to obtain an uniform expansion for the Legendre functions. Previously, this question was analyzed by Thorne in Ref. [8] by using different approach and in Ref. 2] for particular case of the Legendre equation. We would like to note that this special case of calculations plays an important role in the so called functional methods which are at present the most powerful method (see [7]).

The organization of this article is as follows. First of all, in Sec 1 we reobtain the Debay formulas for the uniform expansion of Bessel function by using the WKB approach. In Sec III we apply the same method to the Legendre functions and their derivative. The Appendix contains the list of the first four coefficients in manifest form.

\section{UNIFORM EXPANSION OF THE BESSEL FUNCTIONS}

In this section we reobtain the well-known [1] uniform asymptotic expansion for the Bessel functions of second kind $I_{n}(n \lambda)$ and $K_{n}(n \lambda)$ for large value of $n$.

These functions obey to the following differential equation

$$
W^{\prime \prime}+\frac{1}{\lambda} W^{\prime}=n^{2}\left(1+\frac{1}{\lambda^{2}}\right) W,
$$

where the prime is the derivative with respect $\lambda$.

Let us represent the solution of above equation as a series over small value of $1 / n$ :

$$
W=C e^{n S_{-1}+S_{0}} \sum_{k=0}^{\infty} n^{-k} \omega_{k}
$$

with $\omega_{0}=1$. Using this expression in Eq. (1) we obtain the chain of equations

$$
\begin{aligned}
S_{-1}^{\prime} & =\varepsilon \sqrt{1+\frac{1}{\lambda^{2}}}, \\
S_{0}^{\prime} & =-\frac{1}{2 S_{-1}^{\prime}}\left\{S_{-1}^{\prime \prime}+\frac{1}{\lambda} S_{-1}^{\prime}\right\}, \\
\omega_{k+1}^{\prime} & =-\frac{\varepsilon}{2}\left(\frac{\lambda \omega_{k}^{\prime}}{\sqrt{1+\lambda^{2}}}\right)^{\prime}-\frac{\varepsilon}{8} \frac{\lambda\left(\lambda^{2}-4\right)}{\left(1+\lambda^{2}\right)^{5 / 2}} \omega_{k},
\end{aligned}
$$

where $\varepsilon= \pm 1$ and $k=0,1, \ldots$. With new variable $t=1 / \sqrt{1+\lambda^{2}}$, the last equation may be rewritten in more simple form

$$
\dot{\omega}_{k+1}=\frac{\varepsilon}{2}\left(t^{2}\left(1-t^{2}\right) \dot{\omega}_{k}\right)^{\cdot}+\frac{\varepsilon}{8}\left(1-5 t^{2}\right) \omega_{k},
$$

*Electronic address: nk@dtp.ksu.ras.ru 
where the dot denotes the derivative with respect $t$.

The first integral of the Eqs. (3) has the following form

$$
\begin{aligned}
S_{-1} & =\varepsilon\left(\eta(\lambda)+C_{-1}\right), \\
S_{0} & =-\frac{1}{4} \ln \left(1+\lambda^{2}\right)+C_{0}, \\
\omega_{k+1} & =\frac{\varepsilon}{2} t^{2}\left(1-t^{2}\right) \dot{\omega}_{k}+\frac{\varepsilon}{8} \int_{0}^{t}\left(1-5 t^{\prime 2}\right) \omega_{k}\left(t^{\prime}\right) d t^{\prime}+C_{k+1},
\end{aligned}
$$

where

$$
\eta(\lambda)=\sqrt{1+\lambda^{2}}+\ln \frac{\lambda}{1+\sqrt{1+\lambda^{2}}} .
$$

To find the set of constants $C_{k}, k=-1,0, \ldots$ we take the limit $\lambda \rightarrow \infty$ in our expressions (2), (4) and compare them with well-known asymptotic formulas [1]

$$
I_{n}(n \lambda) \approx \frac{1}{\sqrt{2 \pi n \lambda}} e^{n \lambda}\left(1+O\left(\frac{1}{\lambda}\right)\right), K_{n}(n \lambda) \approx \sqrt{\frac{\pi}{2 n \lambda}} e^{-n \lambda}\left(1+O\left(\frac{1}{\lambda}\right)\right) .
$$

Because of the next term of expansion is $O(1 / \lambda)$ we have to set $C_{k}=0$ for $k \geq 1$. Taking this into account we have the following expression for uniform expansion in the limit $\lambda \rightarrow \infty$ :

$$
W \approx \frac{1}{\sqrt{\lambda}} e^{\varepsilon n \lambda} C e^{\varepsilon n C_{-1}+C_{0}} .
$$

Therefore, $\varepsilon=1$ corresponds to the uniform expansion of $I_{n}(n \lambda)$ and $\varepsilon=-1$ to the $K_{n}(n \lambda)$. For coincidence the expression (7) with the asymptotic expansions (6) we have to set $C_{-1}=C_{0}=0$ and $C=1 / \sqrt{2 \pi n}$ for $\varepsilon=1$, and $C=\sqrt{\pi / 2 n}$ for $\varepsilon=-1$.

Therefore, we arrive at the following well-known formulas for uniform expansion of the Bessel functions

$$
\begin{aligned}
I_{n}(n \lambda) & =\sqrt{\frac{t}{2 \pi n}} e^{n \eta(\lambda)} \sum_{k=0}^{\infty} n^{-k} \omega_{k}(t), \\
K_{n}(n \lambda) & =\sqrt{\frac{\pi t}{2 n}} e^{-n \eta(\lambda)} \sum_{k=0}^{\infty}(-n)^{-k} \omega_{k}(t),
\end{aligned}
$$

where

$$
\omega_{k+1}=\frac{1}{2} t^{2}\left(1-t^{2}\right) \dot{\omega}_{k}+\frac{1}{8} \int_{0}^{t}\left(1-5 t^{\prime 2}\right) \omega_{k}\left(t^{\prime}\right) d t^{\prime} .
$$

In order to find formulas for derivative of the Bessel functions we represent them in the form below

$$
\frac{1}{n} W^{\prime}=\widetilde{C} e^{n \widetilde{S}_{-1}+\widetilde{S}_{0}} \sum_{k=0}^{\infty} n^{-k} \widetilde{\omega}_{k}
$$

Comparing the derivative of Eq. (2) with respect $\lambda$ with above formula we obtain

$$
\begin{aligned}
\widetilde{S}_{-1} & =S_{-1}, \\
\widetilde{S}_{0} & =S_{0}+\ln \left(\varepsilon S_{-1}^{\prime}\right), \\
\widetilde{C} & =\varepsilon C, \\
\widetilde{\omega}_{k} & =\omega_{k}+\frac{\varepsilon}{2} t\left(t^{2}-1\right) \omega_{k-1}+\varepsilon t^{2}\left(t^{2}-1\right) \dot{\omega}_{k-1} .
\end{aligned}
$$

Therefore, with these expressions we arrive at the well-known formulas for uniform expansion of the derivative of the Bessel functions

$$
\frac{1}{n} I_{n}^{\prime}(n \lambda)=\frac{1}{\sqrt{2 \pi n t}} \frac{1}{\lambda} e^{n \eta(\lambda)} \sum_{k=0}^{\infty} n^{-k} \bar{\omega}_{k}(t)
$$




$$
\frac{1}{n} K_{n}^{\prime}(n \lambda)=-\sqrt{\frac{\pi}{2 n t}} \frac{1}{\lambda} e^{-n \eta(\lambda)} \sum_{k=0}^{\infty}(-n)^{-k} \bar{\omega}_{k}(t),
$$

where

$$
\bar{\omega}_{k}=\omega_{k}+\frac{1}{2} t\left(t^{2}-1\right) \omega_{k-1}+t^{2}\left(t^{2}-1\right) \dot{\omega}_{k-1}
$$

\section{UNIFORM EXPANSION OF THE LEGENDRE FUNCTIONS}

In this section we employ the same approach for the Legendre functions. We consider the following equation

$$
\left(1-x^{2}\right) \Psi^{\prime \prime}-2 x \Psi^{\prime}-\left(n^{2} \gamma^{2}+\frac{n^{2}}{1-x^{2}}+2 \xi\right) \Psi=0
$$

which has appeared in context of quantum field theory in curved space-time [5, 6]. Here $x \in(-1,1), n, \gamma$ and $\xi$ are real numbers, and the prime is the derivative with respect $x$. The particular case of this equation for $\xi=1 / 8$ has been considered in Ref. [2].

The solutions of this equation are the Legendre functions first and second kind:

$$
P_{\mu}^{n}[x], Q_{\mu}^{n}[x]
$$

with index

$$
\mu=-\frac{1}{2}+\frac{1}{2} \sqrt{1-8 \xi-4 n^{2} \gamma^{2}}
$$

For $\xi=1 / 8$ these functions are called the cone functions [1].

We assume $n>0$ and consider the following two independent solutions

$$
\begin{aligned}
p_{\mu}^{n}[x] & =P_{\mu}^{-n}[x] \\
q_{\mu}^{n}[x] & =\frac{(-1)^{n}}{2}\left(Q_{\mu}^{n}[x]+Q_{-\mu-1}^{n}[x]\right) \\
& =-\frac{\pi}{2 \sin \pi \mu} P_{\mu}^{n}[-x] .
\end{aligned}
$$

They are real functions for arbitrary $\mu$ and obey the following Wronskian condition

$$
W\left(p_{\mu}^{n}, q_{\mu}^{n}\right)=\frac{1}{1-x^{2}} .
$$

To obtain the uniform expansion of functions (17) for large number $n$ we represent the solution in the WKB form as below

$$
\Psi=C e^{n S_{-1}(x)+S_{0}(x)} \sum_{k=0}^{\infty} n^{-k} \psi_{k}(x)
$$

with $\psi_{0}(x)=1$. We would like to note the difference of the uniform expansion in form (18), which is over inverse degree of $n$, with that considered by Thorne in Ref. [8]. He obtained an expansion over inverse degree of $\mu+1 / 2=$ $\sqrt{1-8 \xi-4 n^{2} \gamma^{2}} / 2$.

Substituting above expression in Eq. (14) we obtain the chain of equations

$$
\begin{aligned}
S_{-1}^{\prime} & =\varepsilon \sqrt{\frac{1}{\left(1-x^{2}\right)^{2}}+\frac{\gamma^{2}}{1-x^{2}}} \\
S_{0}^{\prime} & =-\frac{1}{2}\left\{\frac{S_{-1}^{\prime \prime}}{S_{-1}^{\prime}}-\frac{2 x}{1-x^{2}}\right\} \\
\psi_{1}^{\prime} & =-\frac{1}{2 S_{-1}^{\prime}}\left\{S_{0}^{\prime 2}+S_{0}^{\prime \prime}-\frac{2 x}{1-x^{2}} S_{0}^{\prime}-\frac{2 \xi}{1-x^{2}}\right\},
\end{aligned}
$$




$$
\psi_{k+1}^{\prime}=-\frac{\varepsilon}{2}\left\{\frac{\left(1-x^{2}\right) \psi_{k}^{\prime}}{\sqrt{1+\gamma^{2}\left(1-x^{2}\right)}}\right\}^{\prime}+\psi_{1}^{\prime} \psi_{k}, k \geq 1
$$

where $\varepsilon= \pm 1$.

The first integral of this chain has the following form

$$
\begin{aligned}
S_{-1}(x) & =\varepsilon\left[\gamma \arctan \frac{\gamma x}{\sqrt{1+\gamma^{2}\left(1-x^{2}\right)}}\right. \\
& \left.+\frac{1}{2} \ln \frac{(1+x)\left(1+\gamma^{2}(1-x)+\sqrt{1+\gamma^{2}\left(1-x^{2}\right)}\right)}{(1-x)\left(1+\gamma^{2}(1+x)+\sqrt{1+\gamma^{2}\left(1-x^{2}\right)}\right)}+C_{-1}\right] \\
S_{0}(x) & =-\frac{1}{4} \ln \left(1+\gamma^{2}\left(1-x^{2}\right)\right) \\
\psi_{k+1}(x) & =C_{k+1}(\varepsilon)-\frac{\varepsilon}{2} \frac{1-x^{2}}{\sqrt{1+\gamma^{2}\left(1-x^{2}\right)}} \psi_{k}^{\prime}(x) \\
& +\varepsilon \int_{0}^{x}\left(-\frac{\gamma^{2}}{8}\left[\frac{2-x^{\prime 2}}{\left(1+\gamma^{2}\left(1-x^{\prime 2}\right)\right)^{3 / 2}}-\frac{5 x^{\prime 2}}{\left(1+\gamma^{2}\left(1-x^{\prime 2}\right)\right)^{5 / 2}}\right]\right. \\
& \left.+\frac{\xi}{\left(1+\gamma^{2}\left(1-x^{\prime 2}\right)\right)^{1 / 2}}\right) \psi_{k}\left(x^{\prime}\right) d x^{\prime} .
\end{aligned}
$$

We have already set the constant $C_{0}=0$. This leads to redefinition the constant $C$, only.

The formulas look simpler in terms of new variable

$$
v=\frac{x}{\sqrt{1+\gamma^{2}\left(1-x^{2}\right)}}
$$

instead of $x$. This quantity obeys to inequality: $|v| \leq|x|<1$. In terms of this variable we have

$$
\begin{aligned}
S_{-1}(v) & =\varepsilon\left\{-\frac{1}{2} \ln \frac{1-v}{1+v}+\gamma \arctan \gamma v+C_{-1}\right\} \\
S_{0}(v) & =\frac{1}{4} \ln \frac{1+\gamma^{2} v^{2}}{1+\gamma^{2}} \\
\psi_{k+1}(v) & =C_{k+1}-\frac{\varepsilon}{2} \frac{\left(1-v^{2}\right)\left(1+\gamma^{2} v^{2}\right)}{\left(1+\gamma^{2}\right)} \dot{\psi}_{k}(v) \\
& +\frac{\varepsilon \gamma^{2}}{8\left(1+\gamma^{2}\right)} \int_{0}^{v} d v^{\prime}\left\{5 v^{\prime 2}+\frac{1}{\gamma^{2}}-1+(8 \xi-1) \frac{1+\gamma^{2}}{\gamma^{2}\left(1+\gamma^{2} v^{2}\right)}\right\} \psi_{k}\left(v^{\prime}\right) .
\end{aligned}
$$

In above formulas the dot denotes the derivative with respect new variable $v$.

In order to find constants $C_{k}$ we have to compare our formulas with exact expressions for the Legendre functions at a fixed point. For this reason we take the limit $x \rightarrow 1$ in our formulas

$$
\Psi \approx C\left(\frac{1-x}{2}\right)^{-\varepsilon n / 2} \exp \left[n \varepsilon\left(C_{-1}-\frac{1}{2} \ln \left(\gamma^{2}+1\right)+\gamma \arctan \gamma\right)\right]
$$

and compare them with well-known expressions [3] for the Legendre functions at point $x=1$ :

$$
\begin{gathered}
p_{\mu}^{n}[x]=P_{\mu}^{-n}[x] \approx \frac{1}{n !}\left(\frac{1-x}{2}\right)^{n / 2}, \\
q_{\mu}^{n}[x]=\frac{(-1)^{n}}{2}\left(Q_{\mu}^{n}[x]+Q_{-\mu-1}^{n}[x]\right) \approx \frac{(n-1) !}{2}\left(\frac{1-x}{2}\right)^{-n / 2} .
\end{gathered}
$$

Therefore, from Eqs. (23), (24) we observe that $\varepsilon=-1$ corresponds to $p_{\mu}^{n}[x]$ with $C=1 / n$ !, and $\varepsilon=+1$ corresponds to $q_{\mu}^{n}[x]$ with $C=(n-1) ! / 2$, and

$$
C_{-1}=\frac{1}{2} \ln \left(1+\gamma^{2}\right)-\gamma \arctan \gamma
$$


for both signs of $\varepsilon$. Furthermore, the coefficients $\psi_{k}(v)$ must obey the following condition

$$
\psi_{k}(1)=0 .
$$

Taking into account above formulas we arrive at the following expression for uniform expansion of the Legendre's functions

$$
\begin{aligned}
& p_{\mu}^{n}[x]=\frac{1}{n !}\left[\frac{1+\gamma^{2} v^{2}}{1+\gamma^{2}}\right]^{1 / 4} e^{n S_{-1}(v)} \sum_{k=0}^{\infty} n^{-k} \psi_{k}(v) \\
& q_{\mu}^{n}[x]=\frac{(n-1) !}{2}\left[\frac{1+\gamma^{2} v^{2}}{1+\gamma^{2}}\right]^{1 / 4} e^{-n S_{-1}(v)} \sum_{k=0}^{\infty}(-n)^{-k} \psi_{k}(v),
\end{aligned}
$$

where

$$
\begin{aligned}
S_{-1}(v) & =\frac{1}{2} \ln \frac{1-v}{(1+v)\left(1+\gamma^{2}\right)}-\gamma[\arctan \gamma v-\arctan \gamma] \\
\psi_{k+1}(v) & =\frac{\left(1-v^{2}\right)\left(1+\gamma^{2} v^{2}\right)}{2\left(1+\gamma^{2}\right)} \dot{\psi}_{k}(v) \\
& -\frac{\gamma^{2}}{8\left(1+\gamma^{2}\right)} \int_{1}^{v} d v^{\prime}\left\{5 v^{\prime 2}+\frac{1}{\gamma^{2}}-1+(8 \xi-1) \frac{1+\gamma^{2}}{\gamma^{2}\left(1+\gamma^{2} v^{\prime 2}\right)}\right\} \psi_{k}\left(v^{\prime}\right) .
\end{aligned}
$$

Taking into account the same procedure as we used above for the derivative of the Bessel functions we obtain the following formulas for uniform expansion of the derivative of functions $p_{\mu}^{n}$ and $q_{\mu}^{n}$

$$
\begin{aligned}
\frac{1}{n} \frac{d}{d x} p_{\mu}^{n}[x] & =-\frac{1}{n !}\left[\frac{1+\gamma^{2} v^{2}}{1+\gamma^{2}}\right]^{3 / 4} \frac{1+\gamma^{2}}{1-v^{2}} e^{n S_{-1}(v)} \sum_{k=0}^{\infty} n^{-k} \bar{\psi}_{k}(v) \\
\frac{1}{n} \frac{d}{d x} q_{\mu}^{n}[x] & =\frac{(n-1) !}{2}\left[\frac{1+\gamma^{2} v^{2}}{1+\gamma^{2}}\right]^{3 / 4} \frac{1+\gamma^{2}}{1-v^{2}} e^{-n S_{-1}(v)} \sum_{k=0}^{\infty}(-n)^{-k} \bar{\psi}_{k}(v) \\
\bar{\psi}_{k}(v) & =\psi_{k}(v)-\frac{\gamma^{2} v\left(1-v^{2}\right)}{2\left(1+\gamma^{2}\right)} \psi_{k-1}(v)-\frac{\left(1-v^{2}\right)\left(1+\gamma^{2} v^{2}\right)}{1+\gamma^{2}} \dot{\psi}_{k-1}(v) .
\end{aligned}
$$

The first four coefficients $\psi_{k}$ and $\bar{\psi}_{k}$ are listed in Appendix.

From the recurrent formula (27d) it is possible to find the value of the coefficients $\psi(v)$ for $\gamma \rightarrow \infty$. Indeed, comparing Eq. (27d) in the limit $v \rightarrow 0$ and Eq. (9) in the limit $t \rightarrow 1$ we obtain the following relation

$$
\psi_{k}(0)=(-1)^{k+1} \omega_{k}(1) .
$$

Now we represent formulas obtained in slightly different form which is close to expansion the Bessel functions. We set $x=\cos \epsilon$ and $\gamma=\lambda / \sin \epsilon$ and use the asymptotic expansion for gamma function from Ref. [4]

$$
\begin{aligned}
\ln n ! & =\left(n+\frac{1}{2}\right) \ln n-n+\frac{1}{2} \ln 2 \pi+\sum_{k=1}^{\infty} \frac{B_{2 k}}{2 k(2 k-1)} \frac{1}{n^{2 k-1}}, \\
\ln (n-1) ! & =\left(n-\frac{1}{2}\right) \ln n-n+\frac{1}{2} \ln 2 \pi+\sum_{k=1}^{\infty} \frac{B_{2 k}}{2 k(2 k-1)} \frac{1}{n^{2 k-1}},
\end{aligned}
$$

where $B_{k}$ are the Bernoulli numbers.

With these notations one has

$$
\begin{aligned}
& p_{\mu}^{n}[\cos \epsilon]=\sqrt{\frac{t}{2 \pi n}} e^{n \tilde{\eta}} \sum_{k=0}^{\infty} n^{-k} \psi_{k}^{+}(v)\left(\frac{\sin \epsilon}{\lambda n}\right)^{n}, \\
& q_{\mu}^{n}[\cos \epsilon]=\sqrt{\frac{\pi t}{2 n}} e^{-n \tilde{\eta}} \sum_{k=0}^{\infty}(-n)^{-k} \psi_{k}^{+}(v)\left(\frac{\sin \epsilon}{\lambda n}\right)^{-n}, \\
& \frac{1}{n}{\frac{d p_{\mu}^{n}[x]}{d x}}_{\mid x=\cos \epsilon}=-\sqrt{\frac{1}{2 \pi n t}} e^{n \tilde{\eta}} \sum_{k=1}^{\infty} n^{-k} \bar{\psi}_{k}^{+}(v)\left(\frac{\sin \epsilon}{\lambda n}\right)^{n} \frac{1}{\sin ^{2} \epsilon},
\end{aligned}
$$



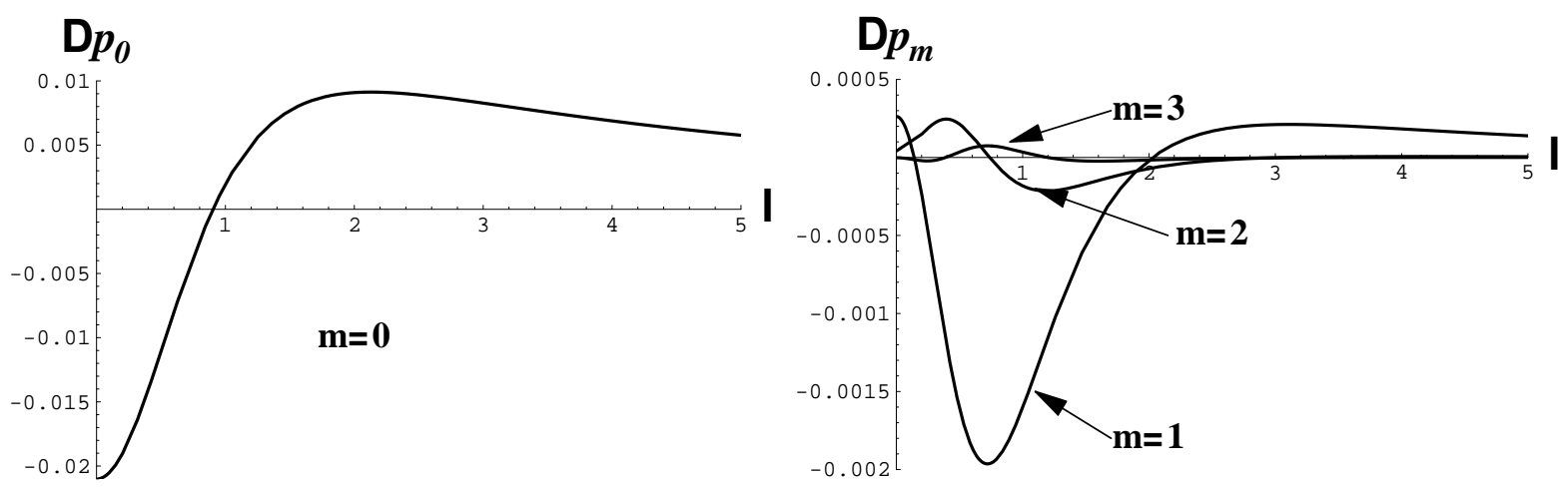

FIG. 1: The plot of the relative errors $\Delta p_{m}=\left(p_{\mu}^{n}-\left(p_{\mu}^{n}\right)_{m}\right) / p_{\mu}^{n}$ versus of $\lambda$ for $\epsilon=0.1, \xi=0$ and $n=4$. Here $\left(p_{\mu}^{n}\right)_{m}$ is the uniform expansion of the Legendre function $p_{\mu}^{n}$ up to degree $m$.

$$
\frac{1}{n}{\frac{d q_{\mu}^{n}[x]}{d x}}_{\mid x=\cos \epsilon}=\sqrt{\frac{\pi}{2 n t}} e^{-n \tilde{\eta}} \sum_{k=1}^{\infty}(-n)^{-k} \bar{\psi}_{k}^{+}(v)\left(\frac{\sin \epsilon}{\lambda n}\right)^{n} \frac{1}{\sin ^{2} \epsilon}
$$

where

$$
\begin{aligned}
\tilde{\eta} & =\ln \frac{\lambda}{\sqrt{1+\lambda^{2}}+\cos \epsilon}-\frac{\lambda}{\sin \epsilon}\left[\arctan \frac{\sin \epsilon}{\lambda}-\arctan \frac{\tan \epsilon}{\lambda t}\right]+1, \\
t & =\frac{1}{\sqrt{1+\lambda^{2}}}, v=t \cos \epsilon \\
\mu & =-\frac{1}{2}+\frac{1}{2} \sqrt{1-8 \xi-\frac{4 n^{2} \lambda^{2}}{\sin ^{2} \epsilon}}
\end{aligned}
$$

and the coefficients $\psi_{k}^{+}$are found from relation

$$
\sum_{k=0}^{\infty} n^{-k} \psi_{k}^{+}(v)=\exp \left(-\sum_{k=1}^{\infty} \frac{B_{2 k}}{2 k(2 k-1) n^{2 k-1}}\right) \sum_{k=0}^{\infty} n^{-k} \psi_{k}(v)
$$

by comparing the same degree of $n$ in the left and right hand sides.

The expressions (30) have the form similar to that for the Bessel functions expansion given by Eq. (8). Furthermore, it is easy to see that in the limit $\epsilon \rightarrow 0$ (argument of the Legendre functions tends to unit and lower index tends to infinity) the uniform expansion obtained is transformed to the uniform expansion of the Bessel functions below

$$
\begin{aligned}
\lim _{\epsilon \rightarrow 0} \mu^{n} p_{\mu}^{n}[\cos \epsilon] & =i^{n} I_{n}(n \lambda), \\
\lim _{\epsilon \rightarrow 0} \mu^{-n} q_{\mu}^{n}[\cos \epsilon] & =i^{-n} K_{n}(n \lambda)
\end{aligned}
$$

as it should be according with well-known formulas $\underline{3}$ ]

$$
\begin{aligned}
\lim _{z \rightarrow \infty} z^{n} P_{z}^{-n}\left[\cos \frac{x}{z}\right] & =J_{n}(x), \\
\lim _{z \rightarrow \infty} z^{n} Q_{z}^{-n}\left[\cos \frac{x}{z}\right] & =-\frac{\pi}{2} Y_{n}(x),
\end{aligned}
$$

where $x=i n \lambda$ and $z=i n \lambda / \epsilon$. In this limit the function $\tilde{\eta}$ given by Eq. (31a) coincides with function $\eta$ (5) in the uniform expansion of Bessel functions:

$$
\lim _{\epsilon \rightarrow 0} \widetilde{\eta}=\ln \frac{\lambda}{\sqrt{1+\lambda^{2}}+1}+\sqrt{1+\lambda^{2}}
$$

The numerical calculation of the relative errors $\Delta p_{m}=\left(p_{\mu}^{n}-\left(p_{\mu}^{n}\right)_{m}\right) / p_{\mu}^{n}$ and $\Delta q_{m}=\left(q_{\mu}^{n}-\left(q_{\mu}^{n}\right)_{m}\right) / q_{\mu}^{n}$ are plotted in Fig. 1 and Fig. 2 for different $m=0,1,2,3$ as function $\lambda$, where $\left(p_{\mu}^{n}\right)_{m}$ and $\left(q_{\mu}^{n}\right)_{m}$ are the uniform expansions of the Legendre functions $p_{\mu}^{n}$ and $q_{\mu}^{n}$ up to degree $n^{-m}$. The difference is smaller the greater $\lambda$. 


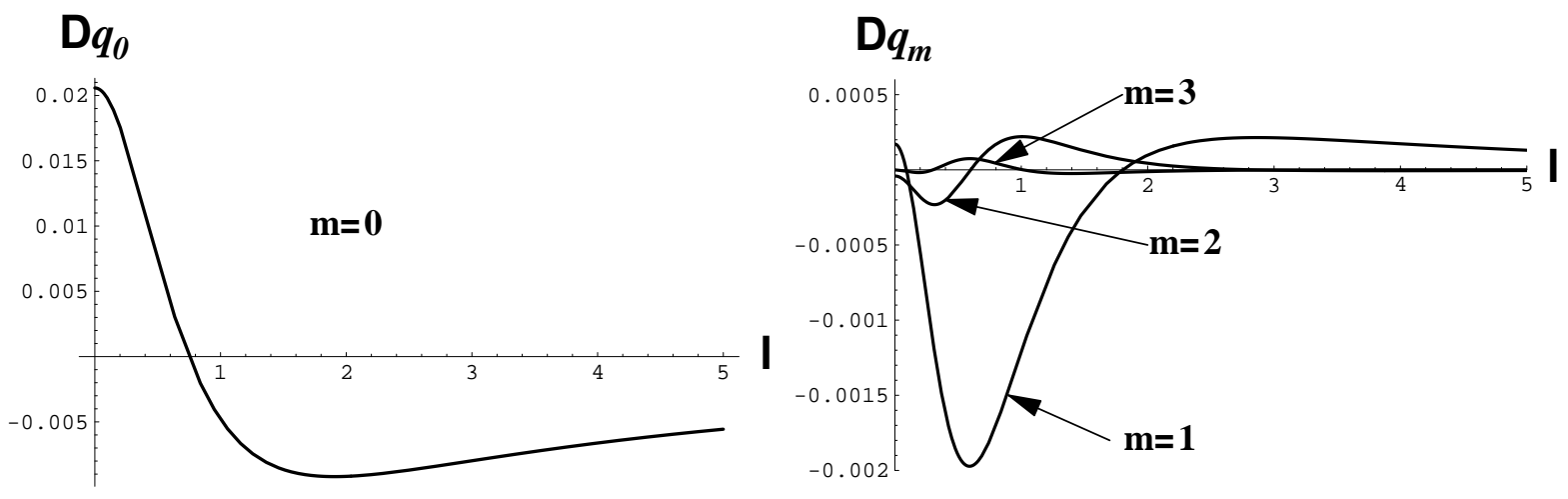

FIG. 2: The plot of the relative errors $\Delta q_{m}=\left(q_{\mu}^{n}-\left(q_{\mu}^{n}\right)_{m}\right) / q_{\mu}^{n}$ versus of $\lambda$ for $\epsilon=0.1, \xi=0$ and $n=4$. Here $\left(q_{\mu}^{n}\right)_{m}$ is the uniform expansion of the Legendre function $q_{\mu}^{n}$ up to degree $m$.

In conclusion we would like to summarize the results. In this paper we obtain the uniform expansion for the Legendre functions $p_{\mu}^{n}[x]$ and $q_{\mu}^{n}[x]$ given by Eq. (17) for large indices $n$ and $\mu=-\frac{1}{2}+\frac{1}{2} \sqrt{1-8 \xi-4 n^{2} \gamma^{2}}$ as a series over inverse degree on $n$. These expansions of the functions are given by Eq. (27) and by Eq. 28) for their derivatives with respect of argument $x$. The coefficients of expansion may be found from recurrent chain of equations (27d) and (28c). The first four coefficients are listed in Appendix.

\section{Acknowledgments}

The author would like to thank Dr. M. Bordag for stimulation of this work and for reading this manuscript. The work was supported by part the Russian Foundation for Basic Research grant N 02-02-17177.

\section{APPENDIX: MANIFEST FORM OF FIRST FOUR COEFFICIENTS.}

Below are the expressions for first four coefficients $\psi_{k}$ and $\bar{\psi}_{k}$ in which we introduced for simplicity the following notations:

$$
\begin{aligned}
\delta=\arctan [\gamma]-\arctan [\gamma v], \zeta=\xi-\frac{1}{8}, v=\frac{x}{\sqrt{1+\gamma^{2}\left(1-x^{2}\right)}} . \\
\psi_{0}=1, \\
\psi_{1}=\frac{\delta \zeta}{\gamma}+\frac{1}{\gamma^{2}+1}\left[\frac{2 \gamma^{2}+3}{24}+\frac{v\left(\gamma^{2}-1\right)}{8}-\frac{5 v^{3} \gamma^{2}}{24}\right], \\
\psi_{2}=\frac{1}{2}\left(\frac{\delta \zeta}{\gamma}\right)^{2}+\frac{\delta \zeta}{\gamma} \frac{1}{\gamma^{2}+1}\left[\frac{2 \gamma^{2}+3}{24}+\frac{v\left(\gamma^{2}-1\right)}{8}-\frac{5 v^{3} \gamma^{2}}{24}\right]+\frac{\zeta\left(-1+v^{2}\right)}{2\left(\gamma^{2}+1\right)} \\
+\frac{1}{\left(\gamma^{2}+1\right)^{2}}\left[\frac{4 \gamma^{4}+84 \gamma^{2}-63}{1152}+\frac{v\left(\gamma^{2}-1\right)\left(2 \gamma^{2}+3\right)}{192}+\frac{v^{2}\left(9 \gamma^{4}-58 \gamma^{2}+9\right)}{128}\right. \\
\left.-\frac{5 v^{3} \gamma^{2}\left(2 \gamma^{2}+3\right)}{576}-\frac{77 v^{4} \gamma^{2}\left(\gamma^{2}-1\right)}{192}+\frac{385 v^{6} \gamma^{4}}{1152}\right], \\
\psi_{3}=\frac{1}{6}\left(\frac{\delta \zeta}{\gamma}\right)^{3}+\frac{1}{2}\left(\frac{\delta \zeta}{\gamma}\right)^{2} \frac{1}{\gamma^{2}+1}\left[\frac{2 \gamma^{2}+3}{24}+\frac{v\left(\gamma^{2}-1\right)}{8}-\frac{5 v^{3} \gamma^{2}}{24}\right]+\frac{\delta \zeta}{\gamma}\left[\frac{1}{\left(\gamma^{2}+1\right)^{2}}\right. \\
\times\left\{\frac{4 \gamma^{4}+84 \gamma^{2}-63}{1152}+\frac{v\left(\gamma^{2}-1\right)\left(2 \gamma^{2}+3\right)}{192}+\frac{v^{2}\left(9 \gamma^{4}-58 \gamma^{2}+9\right)}{128}-\frac{5 v^{3} \gamma^{2}\left(2 \gamma^{2}+3\right)}{576}\right. \\
\left.\left.-\frac{77 v^{4} \gamma^{2}\left(\gamma^{2}-1\right)}{192}+\frac{385 v^{6} \gamma^{4}}{1152}\right\}+\frac{\zeta}{\gamma^{2}+1}\left\{-\frac{2 \gamma^{2}+1}{2 \gamma^{2}}+\frac{v^{2}}{2}\right\}\right]+\frac{\zeta^{2}(1-v)}{2 \gamma^{2}\left(\gamma^{2}+1\right)}
\end{aligned}
$$




$$
\begin{gathered}
+\frac{\zeta}{\left(\gamma^{2}+1\right)^{2}}\left[-\frac{2 \gamma^{2}+7}{48}-\frac{v\left(3 \gamma^{2}-11\right)}{16}+\frac{v^{2}\left(2 \gamma^{2}+3\right)}{48}+\frac{v^{3}\left(44 \gamma^{2}-29\right)}{48}-\frac{35 v^{5} \gamma^{2}}{48}\right] \\
+\frac{1}{\left(\gamma^{2}+1\right)^{3}}\left[-\frac{1112 \gamma^{6}+1116 \gamma^{4}-918 \gamma^{2}+5265}{414720}+\frac{v\left(4 \gamma^{6}+728 \gamma^{4}-4323 \gamma^{2}+711\right)}{9216}\right. \\
+\frac{v^{2}\left(2 \gamma^{2}+3\right)\left(9 \gamma^{4}-58 \gamma^{2}+9\right)}{3072}+\frac{v^{3}\left(2005 \gamma^{6}-37671 \gamma^{4}+37566 \gamma^{2}-2025\right)}{27648} \\
-\frac{77 v^{4}\left(\gamma^{2}-1\right)\left(2 \gamma^{2}+3\right)}{4608}-\frac{13 v^{5} \gamma^{2}\left(1053 \gamma^{4}-3706 \gamma^{2}+1053\right)}{15360}+\frac{385 v^{6} \gamma^{4}\left(2 \gamma^{2}+3\right)}{27648} \\
\left.+\frac{17017 v^{7} \gamma^{4}\left(\gamma^{2}-1\right)}{9216}-\frac{85085 v^{9} \gamma^{6}}{82944}\right] \\
\psi_{0}^{+}=\psi_{0}=1 \\
\psi_{1}^{+}=\psi_{1}-\frac{1}{12}, \\
\psi_{2}^{+}=\psi_{2}-\frac{1}{12} \psi_{1}+\frac{1}{288}, \\
\psi_{3}^{+}=\psi_{3}-\frac{1}{12} \psi_{2}+\frac{1}{288} \psi_{1}+\frac{139}{51840} .
\end{gathered}
$$

$$
\begin{aligned}
& \bar{\psi}_{0}=1 \\
& \bar{\psi}_{1}=\frac{\delta \zeta}{\gamma}+\frac{1}{\gamma^{2}+1}\left[\frac{2 \gamma^{2}+3}{24}-\frac{v\left(3 \gamma^{2}+1\right)}{8}+\frac{7 v^{3} \gamma^{2}}{24}\right] \text {, } \\
& \bar{\psi}_{2}=\frac{1}{2}\left(\frac{\delta \zeta}{\gamma}\right)^{2}+\frac{\delta \zeta}{\gamma} \frac{1}{\gamma^{2}+1}\left[\frac{2 \gamma^{2}+3}{24}-\frac{v\left(3 \gamma^{2}+1\right)}{8}+\frac{7 v^{3} \gamma^{2}}{24}\right]+\frac{\zeta\left(1-v^{2}\right)}{2\left(\gamma^{2}+1\right)} \\
& +\frac{1}{\left(\gamma^{2}+1\right)^{2}}\left[\frac{\left(2 \gamma^{2}-27\right)\left(2 \gamma^{2}-3\right)}{1152}-\frac{v\left(3 \gamma^{2}+1\right)\left(2 \gamma^{2}+3\right)}{192}-\frac{v^{2}\left(15 \gamma^{4}-62 \gamma^{2}+7\right)}{128}\right. \\
& \left.+\frac{7 v^{3} \gamma^{2}\left(2 \gamma^{2}+3\right)}{576}+\frac{v^{4} \gamma^{2}\left(99 \gamma^{2}-79\right)}{192}-\frac{455 v^{6} \gamma^{4}}{1152}\right] \\
& \bar{\psi}_{3}=\frac{1}{6}\left(\frac{\delta \zeta}{\gamma}\right)^{3}+\frac{1}{2}\left(\frac{\delta \zeta}{\gamma}\right)^{2} \frac{1}{\gamma^{2}+1}\left[\frac{2 \gamma^{2}+3}{24}-\frac{v\left(3 \gamma^{2}+1\right)}{8}+\frac{7 v^{3} \gamma^{2}}{24}\right]+\frac{\delta \zeta}{\gamma}\left[\frac{1}{\left(\gamma^{2}+1\right)^{2}}\right. \\
& \times\left\{\frac{\left(2 \gamma^{2}-3\right)\left(2 \gamma^{2}-27\right)}{1152}-\frac{v\left(3 \gamma^{2}+1\right)\left(2 \gamma^{2}+3\right)}{192}-\frac{v^{2}\left(15 \gamma^{4}-62 \gamma^{2}+7\right)}{128}+\frac{7 v^{3} \gamma^{2}\left(2 \gamma^{2}+3\right)}{576}\right. \\
& \left.\left.+\frac{v^{4} \gamma^{2}\left(99 \gamma^{2}-79\right)}{192}-\frac{455 v^{6} \gamma^{4}}{1152}\right\}-\frac{\zeta}{\gamma^{2}+1}\left\{\frac{1}{2 \gamma^{2}}+\frac{v^{2}}{2}\right\}\right]+\frac{\zeta^{2}(1-v)}{2 \gamma^{2}\left(\gamma^{2}+1\right)} \\
& +\frac{\zeta}{\left(\gamma^{2}+1\right)^{2}}\left[\frac{2 \gamma^{2}-1}{48}+\frac{v\left(3 \gamma^{2}-7\right)}{16}-\frac{v^{2}\left(2 \gamma^{2}+3\right)}{48}-\frac{v^{3}\left(44 \gamma^{2}-25\right)}{48}+\frac{35 v^{5} \gamma^{2}}{48}\right] \\
& +\frac{1}{\left(\gamma^{2}+1\right)^{3}}\left[-\frac{1112 \gamma^{6}+5436 \gamma^{4}+1242 \gamma^{2}-1215}{414720}-\frac{v\left(12 \gamma^{6}+904 \gamma^{4}-4281 \gamma^{2}+585\right)}{9216}\right. \\
& -\frac{v^{2}\left(2 \gamma^{2}+3\right)\left(15 \gamma^{4}-62 \gamma^{2}+7\right)}{3072}-\frac{v^{3}\left(2807 \gamma^{6}-42897 \gamma^{4}+37458 \gamma^{2}-1863\right)}{27648} \\
& +\frac{v^{4}\left(99 \gamma^{2}-79\right)\left(2 \gamma^{2}+3\right)}{4608}+\frac{11 v^{5} \gamma^{2}\left(1521 \gamma^{4}-4762 \gamma^{2}+1241\right)}{15360}-\frac{455 v^{6} \gamma^{4}\left(2 \gamma^{2}+3\right)}{27648} \\
& \left.-\frac{385 v^{7} \gamma^{4}\left(51 \gamma^{2}-47\right)}{9216}+\frac{95095 v^{9} \gamma^{6}}{82944}\right]
\end{aligned}
$$


[1] M. Abramowitz and I. Stegun, Handbook of Mathematical Functions, (National Bureau of Standards, U.S. GPO, Washington, D.C., 1964).

[2] A. O. Barvinsky, A. Yu. Kamenshchik and I. P. Karmazin, Ann. Phys. 219, 201 (1992).

[3] H. Bateman and A. Erdélyi, Higher Transcendental Functions. V.1, (Mc Graw-Hill Book Company, Inc, 1953).

[4] H. Bateman and A. Erdélyi, Higher Transcendental Functions. V.2, (Mc Graw-Hill Book Company, Inc, 1953).

[5] N. R. Khusnutdinov and M. Bordag, Phys. Rev. D59, 064017 (1999).

[6] N. R. Khusnutdinov and V. B. Bezerra, Phys. Rev. D64, 083506 (2001).

[7] K. Kirsten, Spectral functions in mathematics and physiscs, (Chapman \& Hall/CRC, Boca Raton, FL, 2001).

[8] R. C. Thorne, Philos. Trans. Roy. Soc. London, 249, 597 (1957). 